\title{
Cajal Bodies and Histone Locus Bodies in Drosophila and Xenopus
}

\author{
Z.F. NiZAMi, S. DeRYUsheva, AND J.G. GALL \\ Carnegie Institution for Science, Baltimore, Maryland 21218 \\ Correspondence: gall@ciwemb.edu
}

\begin{abstract}
The organization of the cell nucleus into specialized compartments is important for nuclear function. We address the significance of compartmentalization by studying the Cajal body, an evolutionarily conserved nuclear organelle proposed to be involved in such diverse functions as assembly of the spliceosome, assembly of the transcription machinery, and modification of spliceosomal small nuclear RNAs. The Cajal body is typically identified by the presence of coilin, a protein of poorly defined function. Here, we demonstrate that coilin is not a unique Cajal body marker but also occurs in a related yet distinct nuclear organelle known as the histone locus body in both Drosophila and Xenopus. We stress the importance of multiple markers not only for identification of nuclear bodies but also for assessing their functional significance.
\end{abstract}

Cajal bodies (CBs) are spherical nuclear organelles that occur in every eukaryote that has been carefully examined, including organisms as evolutionarily divergent as plants, insects, fish, mice, and humans (for review, see Gall 2003; Matera 2006; Stanek and Neugebauer 2006; Morris 2008; Strzelecka et al. 2010). CBs were first observed more than 100 years ago by the neurobiologist Santiago Ramon y Cajal, who described them in neurons of humans and other vertebrates (Lafarga et al. 2009). However, much of our understanding of the function of these bodies has come in the last 20 years, thanks to the availability of new molecular probes and tools. During this time, involvement of the CB in several aspects of RNA processing has been proposed, including modification of spliceosomal small nuclear RNAs (snRNAs), partial assembly of the spliceosome, and assembly of the transcription machinery.

A major advance in the study of the $\mathrm{CB}$ was identification of the protein coilin and cloning of its gene; coilin served as the first molecular marker of the $\mathrm{CB}$ and made the $\mathrm{CB}$ amenable to molecular and genetic analysis (Andrade et al. 1991; Raska et al. 1991). In the intervening years, coilin gained widespread use as the definitive criterion for identifying a nuclear body as a CB (for a while, also known as a coiled body). Here, we present evidence from both Drosophila and Xenopus that coilin can occur in at least two distinct nuclear bodies. One of these contains components of the splicing machinery and is the body most often identified as a CB. The other is a separate nuclear body characterized by the U7 snRNP and other factors involved in histone pre-mRNA processing. We named it the histone locus body (HLB) because of its tight association with histone genes.

We first became aware of the distinction between $\mathrm{CBs}$ and HLBs in our analysis of nuclear bodies in Drosophila. Because Drosophila coilin had not been identi- fied, we looked for CBs with probes against several other components that had previously been assigned to CBs. To our surprise, we found these components distributed between two separate bodies: one that contained U85 small CB-specific RNA (scaRNA), along with U2 snRNA and the survival of motor neuron protein, and a second body that contained the U7 snRNP (Liu et al. 2006). The latter was invariably associated with the histone gene locus and hence was named the HLB. Later, when we identified Drosophila coilin, it became clear that coilin can be present in both the CB and HLB and therefore cannot be considered a signature marker for the CB (Liu et al. 2009).

Our findings in Drosophila prompted us to reconsider earlier descriptions of CBs in the oocyte nucleus of Xenopus and other amphibians. In this chapter, we briefly review studies that clearly linked the Xenopus oocyte bodies to histone transcript processing, well before HLBs were recognized as a distinct cytological entity. We also present data on changes in the morphology and composition of nuclear bodies during oogenesis in both Drosophila and Xenopus. Both organisms begin oogenesis with two types of nuclear bodies: one that contains coilin and one that contains factors involved in histone transcript processing. During oogenesis, the first disappears, whereas the second persists and acquires coilin. Thus, canonical CBs are absent during late stages of oogenesis in both organisms. Instead, the major coilin-containing nuclear organelle is associated with the histone gene locus.

We argue that multiple probes are required for proper identification of CBs and HLBs in other model systems, in part because coilin is not a reliable marker for the CB and in part because different tissues and cell types have different configurations of the two bodies. Finally, we describe recent work using genetic approaches in Drosophila to test the functions of CBs and HLBs. 


\section{MATERIALS AND METHODS}

\section{Drosophila Stocks}

Drosophila melanogaster strains were maintained at $21^{\circ} \mathrm{C}-23^{\circ} \mathrm{C}$ on a standard cornmeal-based medium. A $y$ $w$ stock was used as a control. Coilin-null mutants coil ${ }^{199}$ and $\mathrm{coil}^{203}$ were generated by site-specific mutation using zinc-finger nucleases (Beumer et al. 2008; Liu et al. 2009). The WDR79-null mutant WDR79MB10832 was obtained from the Bloomington stock center.

\section{Immunostaining}

Drosophila tissues were fixed and stained as described previously (Liu et al. 2009). Early-stage Xenopus oocytes were prepared in essentially the same way as Drosophila tissues, with minor modifications. Xenopus laevis ovaries were isolated from $3-5-\mathrm{cm}$ froglets, and oocytes were defolliculated for $2 \mathrm{~h}$ at room temperature with $0.2 \%$ collagenase (Sigma C-6885) in calcium-free OR2 medium. Oocytes were fixed for $10 \mathrm{~min}$ at room temperature in $4 \%$ paraformaldehyde in OR2 medium, then washed and stored in PBS at $4^{\circ} \mathrm{C}$ until staining. Affinity-purified rabbit polyclonal antibodies were generated against Drosophila WDR79 (amino acid residues 136-153) and used at 1:500-1:1000. Rabbit polyclonal antibodies were raised against full-length His-tagged $X$. laevis Lsm10 insoluble protein and used at 1:500-1:1000. Other primary antibodies used were as follows: guinea pig polyclonal serum against $D$. melanogaster coilin used at 1:1000-1:2000 (Liu et al. 2009), rabbit polyclonal serum against $D$. melanogaster Lsm11 used at 1:1000 (Liu et al. 2006), mouse monoclonal antibody $\mathrm{H} 1$ against $X$. laevis coilin used at 1:200 (Tuma et al. 1993), rabbit polyclonal affinity-purified antibody against $D$. melanogaster FLASH used at 1:1000-1:2000 (Yang et al. 2009 [gift from Z. Dominski]), mouse monoclonal antibody against human symplekin used at 1:1000 (BD Transduction Laboratories), mouse monoclonal antibody ARNA-3 against the largest subunit of RNA polymerase (Pol) II (RPB1) used at 1:500 (Research Diagnostics), mouse monoclonal antibody MPM-2 used at $2 \mu \mathrm{g} / \mathrm{mL}$ (Upstate Biotechnology), and rabbit polyclonal affinity-purified antibody against phosphohistone $\mathrm{H} 3$ used at 1:100 (Upstate Biotechnology).

\section{FISH}

Fluorescence in situ hybridization (FISH) for Drosophila and Xenopus whole-mount tissues was performed as described by Liu et al. (2009), using fluorescently labeled probes. FISH for transcripts from the histone gene cluster of Notophthalmus viridescens was performed with a biotinylated probe against a 225-bp satellite DNA sequence, which occurs as a spacer between adjacent histone gene clusters (Stephenson et al. 1981).

\section{Fluorescent Primer Extension Analysis}

2'-O-methylation of spliceosomal snRNAs was mapped using the fluorescent primer extension assay described below (Deryusheva and Gall 2009). Total RNA was isolated from $y w$, coilin-null, and WDR79-null flies.

\section{RESULTS AND DISCUSSION}

\section{Coilin in the CB and the HLB in Drosophila and Xenopus}

Our recent observation that antibodies against Drosophila coilin can label both CBs and HLBs in the same nucleus provided cogent evidence that coilin may not be a reliable marker for the CB (Liu et al. 2009). Furthermore, we found that certain cell types that lack CBs may nonetheless contain bright coilin-positive HLBs. These relationships are particularly apparent during Drosophila oogenesis, which follows the stereotyped pattern outlined in Figure 1A. Each ovariole of the ovary consists of a single row of egg chambers of increasing age and size along the anterior-to-posterior axis; egg chambers consist of a germline component (15 nurse cells and one oocyte) and a somatic component (a sheet of follicle cells encapsulating the germline). Nurse cell nuclei from early-stage egg chambers usually contain single CBs and HLBs (Fig. 1B,E), with coilin at a high level only in the CB (Fig. 1B). Two major changes are observable by the mid stages of oogenesis: First, the single $\mathrm{CB}$ is replaced by multiple smaller bodies (Fig. 1C,F) and second, coilin becomes evident in the HLBs as well as in the CBs (Fig. 1C). These changes take place at approximately the same time in all nurse cell nuclei within an egg chamber, although nuclei at the anterior of the chamber lag somewhat behind the more posterior nuclei (next to the oocyte). Finally, near the end of oogenesis, CBs disappear from the nurse cell nuclei (Fig. $1 \mathrm{D}, \mathrm{G})$, whereas the HLBs remain as prominent as in earlier stages and now contain a high level of coilin (Fig. 1D).

During maturation of the egg chamber, nurse cell nuclei become highly polyploid by undergoing multiple rounds of DNA replication without nuclear or cell division. As the nuclei alternate between the $\mathrm{G}$ and $\mathrm{S}$ phase of the cell cycle, the composition of the HLBs also undergoes cyclical changes. To determine whether the presence of coilin in HLBs was cell cycle regulated, we immunostained egg chambers for both coilin and an antigen known as MPM2, which is a marker for the S phase (Calvi et al. 1998; White et al. 2007). Within a given egg chamber, all HLBs stained equally for coilin, whereas only a subset labeled with the anti-MPM-2 antibody. Thus, the appearance of coilin in the HLB is related to the developmental age of the egg chamber, not to the specific stage in the cell cycle.

Having found such dynamic changes in the CBs and HLBs during Drosophila oogenesis, we decided to reexamine nuclear bodies in the Xenopus oocyte. The amphibian oocyte nucleus or germinal vesicle $(\mathrm{GV})$ has long been a favorite subject for study. Its large size permits it to be hand-isolated, and it contains exceptionally large and transcriptionally active lampbrush chromosomes (Callan 1986). Most studies on Xenopus have concentrated on the late stages of oogenesis (0.8-1.2-mm diameter oocytes), at which time the GV contains 50-100 extrachromosomal bodies originally called spheres on the basis of their shape (Callan 1986). With the discovery that coilin is a prominent component of spheres (Tuma et al. 1993; Wu et al. 1994), these structures were renamed coiled bodies and then CBs (Gall et al. 1999), and these names have been used in numerous subsequent publications. One feature that seemed 


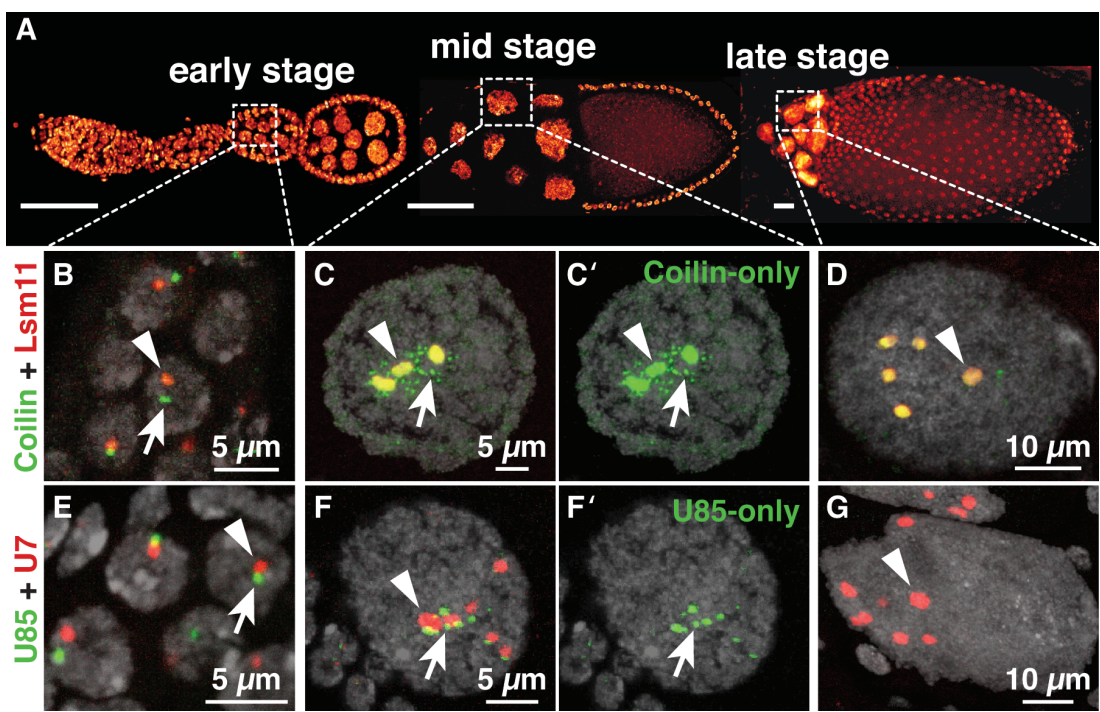

Figure 1. CB and HLB distribution during Drosophila oogenesis. (A) Drosophila ovariole stained with DAPI. Boxes surround nurse cell nuclei from early-, mid-, and late-stage egg chambers. Scale bars, $20 \mu \mathrm{m}$. $(B-D)$ Nurse cell nuclei from early $(B)$, mid $(C)$, and late $(D)$ stages of oogenesis immunostained for coilin (green) and Lsm11 (red). (Gray) DAPI stain. ( $\left.C^{\prime}\right)$ Coilin pattern from $(C)$. ( $E-$ $G$ ) Nurse cell nuclei from early $(E)$, mid $(F)$, and late $(G)$ stages of oogenesis after FISH for U85 scaRNA (green) and U7 snRNA (red). $\left(\mathrm{F}^{\prime}\right)$ U85 pattern from $(F)$. Arrowheads point to HLBs, arrows to CBs. Early-stage nurse cells have coilin in the CB. Mid-stage nurse cells have coilin in the CB and the HLB. Late-stage nurse cells do not have CBs; coilin is high in the HLB.

to set these oocyte CBs apart from CBs found in mammalian tissue culture and elsewhere was their association with histone genes and histone transcript processing. Although most of the oocyte bodies are free in the nucleoplasm, a few are attached to the lampbrush chromosomes at the histone gene loci (Gall et al. 1981; Callan et al. 1991), making them HLBs by definition (Fig. 2). Furthermore, a variety of factors involved in histone transcript processing are found in them. U7 snRNA was the first such factor to be demonstrated (Wu and Gall 1993) and is, in fact, more abundant than the splicing snRNAs (Bellini and Gall 1998). Another histone processing factor that occurs in these bodies is the stem-loop-binding protein (SLBP) (Abbott et al. 1999), and more recently, we have demonstrated the presence of symplekin and FLASH.

Given all of these data pointing to an association of the oocyte bodies with histone genes and histone transcript processing, it is difficult to escape the conclusion that they should be considered HLBs and not typical CBs. An ex- amination of Xenopus oocytes throughout oogenesis underscores this conclusion and provides striking parallels with the events that take place during Drosophila oogenesis. We have examined early-stage Xenopus oocytes as whole mounts, using antibodies and FISH probes for $\mathrm{CB}$ and HLB components. In oocytes up to about $100 \mu \mathrm{m}$ in diameter, coilin does not colocalize with HLB markers such as FLASH and U7 snRNA, but instead occurs in separate ring-like or crescent-shaped bodies (Fig. 3A). These early-stage coilin-positive bodies have not yet been fully characterized, but like the Drosophila CBs, they are no longer apparent at later stages of oogenesis (Fig. 3B). The bodies that label with HLB markers in early-stage Xenopus oocytes are negative for coilin relative to the nucleoplasmic level (Fig. 4A). Mid-stage oocytes (100-300- $\mu \mathrm{m}$ diameter) are difficult to study in the intact ovary, so we digested ovary fragments with collagenase and examined individual defolliculated oocytes as whole mounts. As the oocyte increases in size, the HLBs show progressive en-

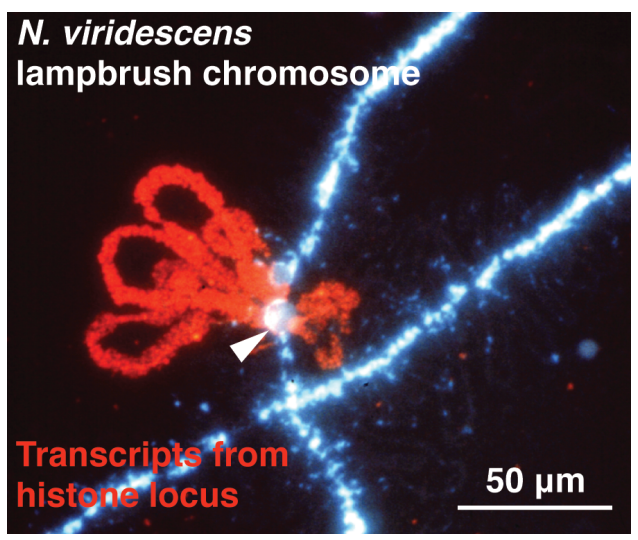

Figure 2. Histone gene locus on a lampbrush chromosome from an oocyte of the newt Notophthalamus viridescens. The DNA axis of the lampbrush chromosome is labeled with DAPI (blue and white). Transcripts from the histone gene locus are labeled with a FISH probe against the spacer region of the histone gene cluster (red). At the base of the transcription loops are two unlabeled HLBs attached to the DNA axis on the chromosome (arrowhead). The HLBs do not contain histone transcripts. 

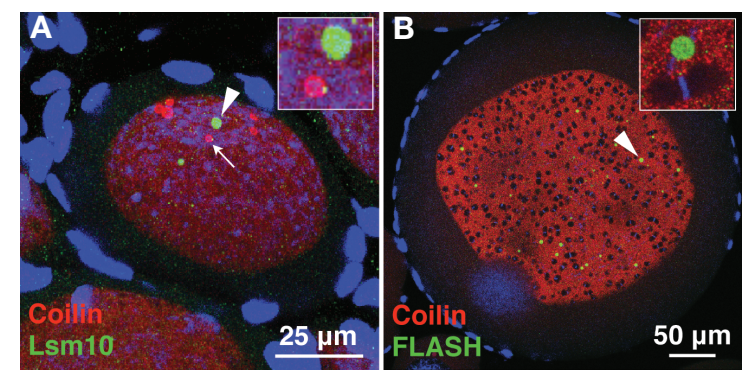

Figure 3. Early-stage Xenopus oocytes immunostained for coilin and HLB components. (A) $100-\mu \mathrm{m}$ Xenopus oocyte immunostained for coilin (red) and Lsm10 (green). DNA is stained with DAPI (blue). The oocyte has a large nucleus with a high concentration of coilin in the nucleoplasm; it is surrounded by a layer of follicle cells. There are two types of nuclear bodies at this stage; one contains Lsm10, a component of the HLB (arrowhead), and the other contains coilin (arrow). (Inset) The two types of body at higher magnification. (B) 400- $\mu \mathrm{m}$ Xenopus oocyte immunostained for coilin (red) and FLASH (green). DNA is stained with DAPI (blue). Coilin is present in the nucleoplasm of the oocyte nucleus but is no longer enriched in discrete bodies. HLBs are stained with the antibody against FLASH. (Inset) HLB at higher magnification and one of the numerous extrachromosomal nucleoli, each of which contains a rod-like mass of rDNA (DAPI, blue).

richment of coilin relative to the nucleoplasm (Fig. 4B,C). With the exception of the increase in coilin concentration, their composition does not change for other HLB components that we have examined, including the U7 snRNPspecific protein Lsm10 (Fig. 5A), FLASH (Fig. 5B), and
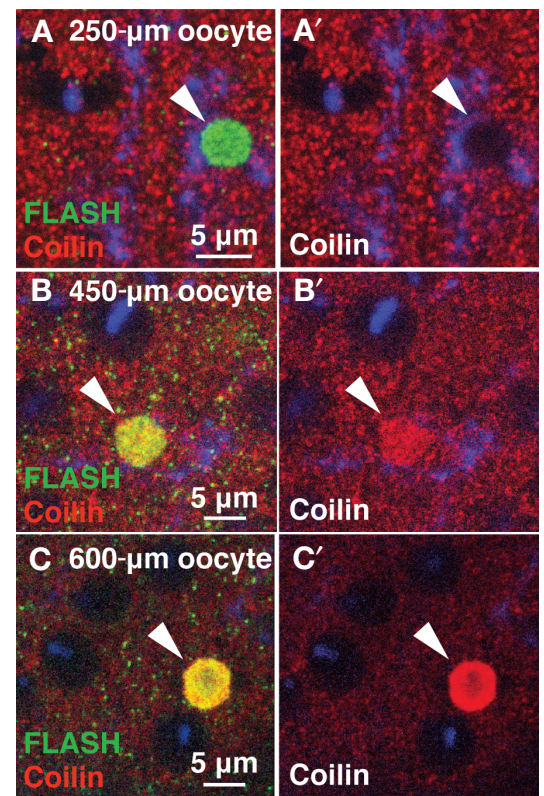

Figure 4. HLBs from progressively older oocytes of Xenopus, showing increasing concentration of coilin. $(A-C)$ Enlarged regions of nuclei from early-, mid-, and late-stage Xenopus oocytes labeled with antibodies against coilin (red) and FLASH (green). DNA is stained with DAPI (blue). Arrowheads point to HLBs. Extrachromosomal nucleoli are unstained except for rDNA (rodlike DAPI label). $\left(A, A^{\prime}\right) 250-\mu \mathrm{m}$ oocyte with HLBs that lack coilin. $\left(B, B^{\prime}\right) 450-\mu \mathrm{m}$ oocyte with HLBs that contain a slight enrichment of coilin relative to the nucleoplasmic level. $\left(C, C^{\prime}\right) 600$ $\mu \mathrm{m}$ oocyte with HLBs that contain significant enrichment of coilin relative to the nucleoplasmic level. symplekin (Fig. 5C). Thus, as in Drosophila, late-stage Xenopus germline nuclei are characterized by the absence of typical CBs and the presence of HLBs that contain a high concentration of coilin.

\section{Transcription Machinery and the HLB}

The realization that the GV bodies in Xenopus are HLBs has some immediate implications. First, we are now better able to reconcile data from mammalian CBs, which have focused on spliceosomal snRNAs, and from Xenopus HLBs, which contain only minimal levels of spliceosomal snRNAs (Gall et al. 1999). Second, we can use what we know about the Xenopus HLB to learn more about the Drosophila HLB and vice versa. For example, several previous studies on late-stage Xenopus GVs demonstrated that HLBs are enriched for components of the transcription machinery, such as basal transcription factors and various subunits of all three RNA polymerases (Gall et al. 1999; Morgan et al. 2000; Doyle et al. 2002; Murphy et al. 2002). We therefore used some of the markers from these studies to examine whether a similar enrichment occurs in Drosophila HLBs. Using the ARNA-3 antibody, which was raised against a conserved epitope in the largest subunit of RNA Pol II, we observe an appreciable enrichment in Drosophila nurse cell HLBs compared to the nucleoplasm (Fig. 6A). The ARNA-3 antibody only labels HLBs in a subset of nuclei in a given egg chamber, suggesting that its localization is cell cycle dependent. We confirmed this suggestion by showing colocalization of the ARNA-3 and MPM-2 label (Fig. 6B). The implication is that Pol II is more highly concentrated in HLBs during $\mathrm{S}$ phase, when histone transcription is maximal (Marzluff 2005). HLBs in early-stage Xenopus oocytes can also be labeled with the ARNA-3 antibody, providing further confirmation that their composition is similar to that of latestage HLBs (Fig. 6C).

An important issue is the relationship between the HLB and the histone gene locus with which it is associated. An analogy that comes immediately to mind is the nucleolus and the rDNA locus. In this case, the rDNA genes are an integral part of the body, with transcription as well as processing and packaging of the transcripts taking place inside the nucleolus itself (DiMario 2004; Raska et al. 2006). The Balbiani rings of Chironomus represent a similar, somewhat less extreme, case where the "body" consists of multiple transcription loops with associated transcription machinery, nascent transcripts, processing factors, and completed RNP particles ready for export to the cytoplasm (Daneholt 2001). Despite the superficial similarity, we believe that HLBs are fundamentally different from nucleoli and Balbinai rings in their relationship to the chromosomal locus with which they are associated. The amphibian GV provides the clearest evidence. The GV contains numerous extrachromosomal nucleoli and HLBs. As has been known for many years, the nucleoli contain their own extrachromosomal copies of rDNA (Fig. 4) and actively transcribe rRNA (Brown and Dawid 1968; Gall 1968; Miller and Beatty 1969). In other words, a nucleolus and an active rDNA locus are the same thing, even 

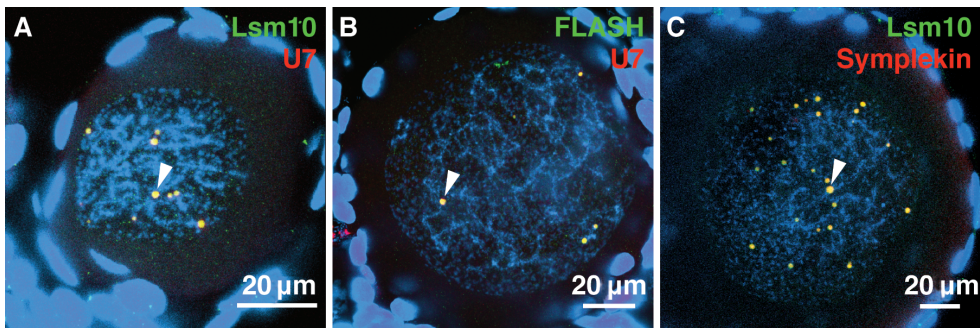

Figure 5. HLB components in early-stage Xenopus oocytes. In each panel, colocalization of the red and green probes results in yellow HLBs. DNA is stained with DAPI (blue). (A) Lsm10 antibody stain (green), U7 antisense FISH (red). (B) FLASH antibody stain (green), U7 antisense FISH (red). (C) Lsm10 (green), symplekin (red) antibody stains.

when not part of a chromosome. HLBs are different. Extrachromosomal HLBs do not contain histone genes and so by definition cannot transcribe histone pre-mRNA. More importantly, they do not contain detectable histone transcripts. One could argue that this is because the GV is in $\mathrm{G}_{2}$ of the cell cycle when histone transcripts are not normally made. But the oocyte is an exception, because it transcribes histone genes and even stores histones in the cytoplasm during the extended prophase of oogenesis (for review, see Stein et al. 1984). The relationship of attached HLBs to transcription at the histone gene loci is particularly instructive. This has been studied extensively in the newt Notophthalmus (Gall et al. 1983), with the overall conclusion that transcription occurs on lampbrush loops associated with HLBs, but neither histone genes nor transcripts are in the HLB itself (Fig. 2). Although these data
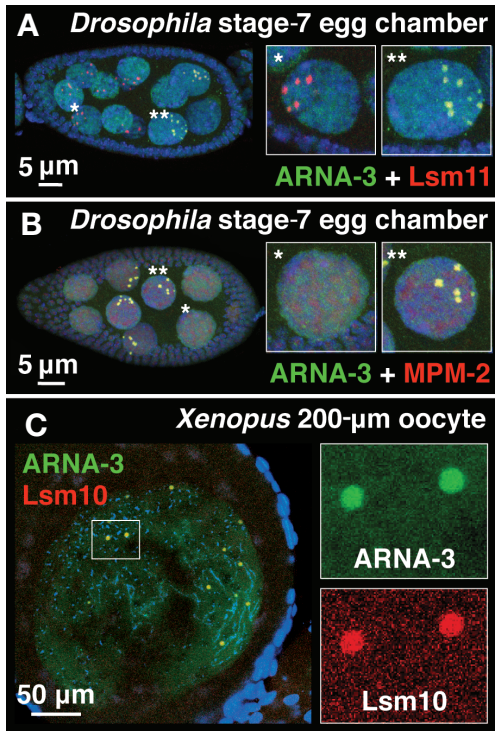

Figure 6. RNA Pol II in the HLB of Drosophila and Xenopus. (A) Mid-stage Drosophila egg chamber stained with ARNA-3 antibody against Pol II (green) and with Lsm11 antibody (red). DNA is stained with DAPI (blue). Insets show starred nurse cells at higher magnification. All nurse cell nuclei contain HLBs that stain for Lsm11, but only a subset of nuclei have HLBs enriched with the ARNA-3 label (two-star nurse cell). (B) Mid-stage Drosophila egg chamber labeled with ARNA-3 antibody (green) and MPM2 , an antibody that labels HLBs in S phase of the cell cycle (red). DNA is stained with DAPI (blue). ARNA-3 colocalizes with MPM-2 label in HLBs from S-phase nuclei (yellow label in the two-star nucleus). HLBs in nuclei that are not in S phase do not label (unstained one-star nucleus). (C) Early-stage Xenopus oocyte labeled with ARNA-3 antibody (green) and Lsm10 antibody (red) (Insets) Single-channel images of two HLBs in which ARNA-3 and the Lsm10 stain are colocalized. show conclusively that HLBs in the amphibian oocyte are not hypertrophied gene loci like the nucleolus and Balbiani rings, they leave unanswered several major questions about the biogenesis and function of HLBs. Do HLBs form at the histone gene locus and detach to become free bodies? Or do HLBs form de novo in the nucleoplasm and later travel to the locus? Where are the actual sites of histone transcript processing and how do the processing factors in the HLBs get there? Although the HLBs of Drosophila are superficially quite different from those in the amphibian oocyte, they pose some of the same questions. To determine the physical relationship between the gene locus and the HLBs will be more difficult in Drosophila because of the much smaller size and the fact that the HLBs seem to be invariably associated with the locus. Super resolution microscopy could be of some help, as would careful examination of the situation in the giant polytene nuclei. There is already circumstantial evidence that histone gene transcription is not required for formation or maintenance of HLBs. This follows from the observation that HLBs are present throughout the interphase, but contain detectable levels of Pol II only during $\mathrm{S}$ phase, when histone transcription occurs (Fig. 6A,B)

\section{Drosophila Mutants that Lack a CB}

We previously described two Drosophila coilin-null mutants that lack CBs but are nonetheless viable and fertile (Liu et al. 2009). The fact that these mutant flies had no obvious deleterious phenotype was an immediate indication that one of the commonly postulated functions for the $\mathrm{CB}$ was not likely to be true, namely, that the $\mathrm{CB}$ is the exclusive site for posttranscriptional modification of spliceosomal snRNAs (Jády et al. 2003). In subsequent experiments, we used a primer extension assay to demonstrate that spliceosomal snRNAs are indeed properly modified in coilin-null flies (Deryusheva and Gall 2009). Since completing that study, we have identified a second mutant that lacks CBs: a null for a different CB component, WDR79. WDR79 is a WD-repeat protein that binds to a conserved sequence in scaRNAs and is required for proper localization of scaRNAs to the CB (Tycowski et al. 2009; Venteicher et al. 2009). The cellular localization of WDR79 is similar to that of coilin, as shown by antibody staining. Thus, WDR79 is concentrated in all CBs that we have examined and in some HLBs, such as those in late-stage nurse cell nuclei. As in the Drosophila coilin-null mutants, cells from WDR79-null flies do not contain detectable CBs (Fig. 7G-I). Nevertheless, they have the correct posttranscriptional modifications on their spliceosomal snRNAs; an example is shown in the 

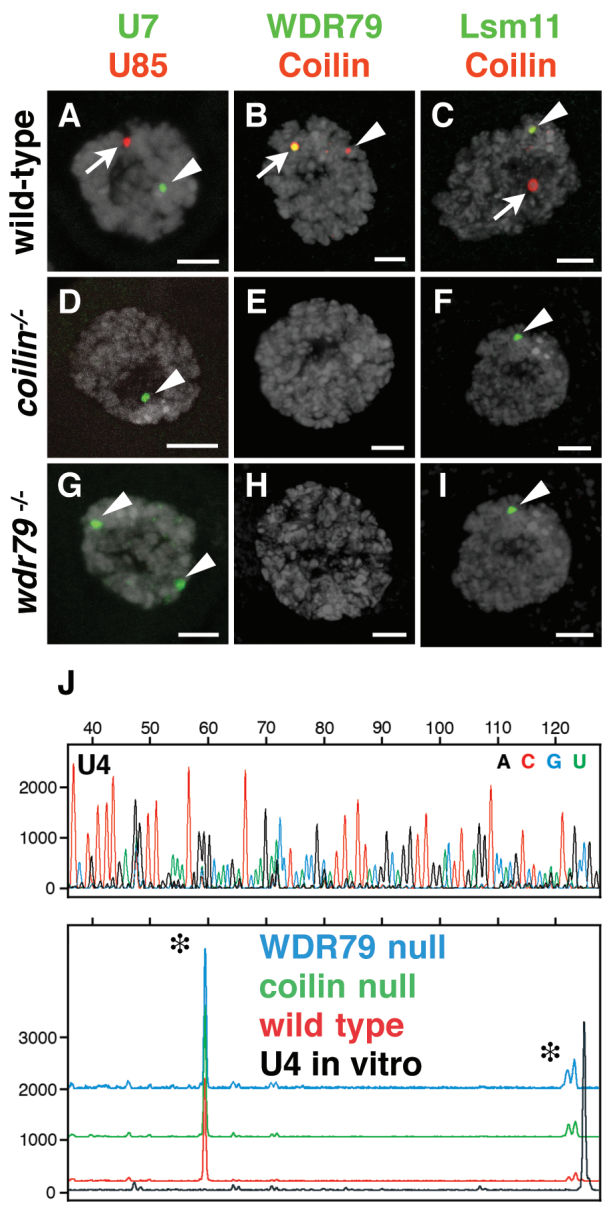

Figure 7. Drosophila CB mutants have normal HLBs and spliceosomal snRNA modifications. ( $A-I)$ Malpighian tubule nuclei from wild-type $(A-C)$, coilin-null $(D-F)$, and WDR79-null $(G-I)$ flies labeled with DAPI (gray) and markers for HLBs (arrowheads) and CBs (arrows). ( $A, D, G)$ FISH for U7 snRNA (green) and U85 scaRNA (red). $(B, E, H)$ Antibody stain for WDR79 (green) and coilin (red). (C,F,I) Antibody stain for Lsm11 (green) and coilin (red). WDR79-null mutants are similar to coilin-null mutants; they lack CBs but have normal HLBs. Scale bar, $5 \mu \mathrm{m}$. (J) Primer extension assay to detect posttranscriptional $2^{\prime}$ - $O$-methyl modifications on the U4 spliceosomal snRNA in wild-type and mutant flies. The peaks (asterisks) represent positions where the primer extension reaction terminated ( 1 nucleotide downstream from the 2'-O-methyl group). The peaks for RNA from wild-type flies (red) and CB mutant flies (green and blue) overlap; for ease of viewing, the individual curves have been shifted on the $y$ axis. In vitrotranscribed U4 snRNA (black) is not modified and has no peaks.

primer extension analysis of U4 spliceosomal snRNA (Fig. 7J). The results with both coilin-null and WDR79-null flies show that guide ribonucleoproteins (RNPs) can modify their substrates outside of a cytologically detectable CB, presumably in RNA-protein complexes in the nucleoplasm that are below the detection limit of the light microscope. The question remains as to why there is a CB at all. Possibly, a larger body leads to more efficient modification reactions, by either mass action or allowing local recycling of components, as would be consistent with results from mathematical models of U4 and U6 di-snRNP assembly (Klingauf et al. 2006).
Alternatively, CBs might serve to sequester certain components, thus negatively regulating the progression of these reactions. In either case, it is obvious that there are no gross physiological phenotypes for Drosophila CB mutants under laboratory conditions, and it may be necessary to test these hypotheses under conditions of stress.

The colocalization of coilin and WDR79, along with the similarity of their null phenotypes, suggests that the two proteins may exist in the cell in a molecular complex. Because WDR79 binds to the CAB box of scaRNAs (Tycowski et al. 2009; Venteicher et al. 2009), it is possible that coilin and WDR79 function together to target scaRNAs to the CB. This hypothesis remains to be tested. Even if true, it would not explain the colocalization of coilin and WDR79 in the HLB, which lacks scaRNAs. Still another puzzle is the persistence of HLBs in the absence of CBs in mutants that are null for either coilin or WDR79. Both proteins occur in both bodies, sometimes within the same nucleus, as in nurse cells from mid-stage egg chambers. Thus, the integrity of the $\mathrm{CB}$, but not the HLB, is compromised by the lack of either protein, suggesting that the pool of coilin and WDR79 may have different functions in the two bodies.

\section{CB and HLB Distribution in Different Drosophila Tissues and Cell Types}

Because coilin is not a unique marker for the $\mathrm{CB}$, it is important to use multiple probes in assessing nuclear bodies in unfamiliar tissues. In addition to immunostaining with probes against coilin and other common CB components, we find that in situ hybridization with probes against the U7 snRNA and any of the abundant scaRNAs (such as U85) is helpful in distinguishing CBs from HLBs. In all cases so far examined, these signals do not colocalize (Fig. 1F, F'), but when there is doubt, in situ hybridization against the histone gene locus itself will, by definition, identify the HLB. In Drosophila, we have not yet found nuclei in which HLBs exist separately from the histone locus, as in the amphibian oocyte. In Drosophila, the most common configuration in diploid cells and those with a low level of polyploidy is one CB and one HLB per nucleus. Somatic pairing probably accounts for the fact that two or more HLBs per nucleus are unusual. Nevertheless, multiple HLBs occur in nurse cell nuclei (Fig. 1C,D; Fig. 6A,B), which contain a polyploid set of polytene chromosomes after stage 5 (Dej and Spradling 1999). Multiple CBs occur in these same nuclei as well as in the giant salivary gland nuclei of third instar larvae. However, so far we have no evidence that CBs are associated with specific gene loci in Drosophila. A significant exception to the one CB plus one HLB configuration for diploid nuclei is found in undifferentiated eye imaginal disk cells and larval neuroblasts, which have an HLB but no CB (Fig. 8A). In contrast to somatic nuclei, both male and female germline nuclei display a diverse array of configurations. Male and female germline stem cells lack CBs but have HLBs (Fig. 8C), and in this respect, they resemble undifferentiated eye imaginal disk cells and neuroblasts (Fig. 8A). Spermatocyte, spermatid, and sperm nuclei lack 

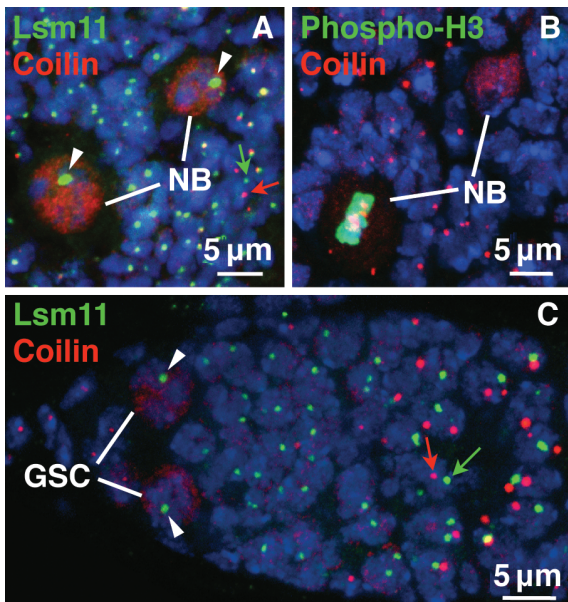

Figure 8. Some undifferentiated cells in Drosophila have HLBs but lack CBs. $(A)$ Third instar larval brain immunostained for Lsm11 (green) and coilin (red). DNA is stained with DAPI (blue). Like germline stem cells, neuroblasts (NB) have an HLB (arrowheads) but no $\mathrm{CB}$, despite a high level of diffuse nucleoplasmic coilin. Most surrounding nuclei also have HLBs without CBs, but a few have both CBs (red arrow) and HLBs (green arrow). (B) Third instar larval brain immunostained for coilin (red) and phosphohistone $\mathrm{H} 3$, a marker for mitotic nuclei (green). One of the two neuroblasts (NB) is dividing and has green chromosomes, whereas the other is not dividing and has diffuse coilin stain in the nucleoplasm (red). (C) Germarium at the tip of a Drosophila ovariole immunostained for Lsm11 (green) and coilin (red). DNA is stained with DAPI (blue). The two germline stem cells (GSCs) each have an HLB (arrowheads) but no CB, despite the relatively high coilin level in the nucleoplasm. Differentiated daughter cells have both CBs (red arrow) and HLBs (green arrow).

CBs, although they have unusually high levels of diffuse coilin in the nucleoplasm (Liu et al. 2009). The significance of these variations in nuclear bodies remains obscure.

Given the variety of CB and HLB configurations in Drosophila tissues, as well as the variable levels of coilin in HLBs, it seems possible that the coilin-positive bodies reported in the literature for other model systems may be a mixture of CBs and HLBs. Indeed, this question has already been addressed in regard to the classification of mammalian HLBs, which label with FLASH, and the histone gene-specific transcription factor NPAT (BongiornoBorbone et al. 2008). The analysis concentrated on coilin, which colocalized at $\mathrm{S}$ phase with HLBs, defined as bodies associated with histone gene loci. Although this colocalization was reasonably interpreted as a merging of HLBs and $\mathrm{CBs}$, it would be interesting to look for other CB markers, such as the scaRNAs. Broadly speaking, it would also be interesting to assess whether mammalian systems, as in Drosophila, have different CB and HLB configurations depending on cell type and differentiation state. This possibility is supported by a recent study showing altered nuclear body composition during the course of differentiation in a human neuroblastoma cell line (Clelland et al. 2009). Finally, it is important to consider the possibility that there may be additional coilin-positive bodies that are distinct from "traditional" CBs, i.e., those involved with
snRNP maturation. For example, it is not yet clear how we should classify the coilin-positive bodies in early-stage Xenopus oocytes. And CBs of Arabidopsis differ from canonical vertebrate $\mathrm{CBs}$ in that they are implicated in small RNA processing pathways ( $\mathrm{Li}$ et al. 2006).

\section{CONCLUSION}

Here, we have discussed evidence from Drosophila and Xenopus demonstrating that coilin is present in at least two distinct types of nuclear bodies, CBs and HLBs. Thus, coilin cannot be used as a unique marker for the CB. Based on our studies, we conclude that the coilin-positive bodies in late-stage Xenopus oocytes should be considered HLBs, not CBs. Having now characterized CBs and HLBs in both Drosophila and Xenopus, we are using a combination of genetic and cell biological approaches to evaluate various models of CB and HLB function.

\section{ACKNOWLEDGMENTS}

We thank Ji-Long Liu for his input in the early stages of this investigation. Zheng' an Wu kindly supplied Figure 2. Research is supported in part by National Institutes of Health grant GM-33397 to J.G.G., an American Cancer Society Professor of Developmental Genetics.

\section{REFERENCES}

Abbott J, Marzluff WF, Gall JG. 1999. The stem loop binding protein (SLBP1) is present in coiled bodies of the Xenopus germinal vesicle. Mol Biol Cell 10: 487-499.

Andrade LEC, Chan EKL, Raska I, Peebles CL, Roos G, Tan EM. 1991. Human autoantibody to a novel protein of the nuclear coiled body: Immunological characterization and cDNA cloning of p80-coilin. J Exp Med 173: 1407-1419.

Bellini M, Gall JG. 1998. Coilin can form a complex with the U7 small nuclear ribonucleoprotein. Mol Biol Cell 9: 2987-3001.

Beumer KJ, Trautman JK, Bozas A, Liu JL, Rutter J, Gall JG, Carroll D. 2008. Efficient gene targeting in Drosophila by direct embryo injection with zinc-finger nucleases. Proc Natl Acad Sci 105: 19821-19826.

Bongiorno-Borbone L, De Cola A, Vernole P, Finos L, Barcaroli D, Knight RA, Melino G, De Laurenzi V. 2008. FLASH and NPAT positive but not coilin positive Cajal bodies correlate with cell ploidy. Cell Cycle 7: 2357-2367.

Brown DD, Dawid IB. 1968. Specific gene amplification in oocytes. Science 160: 272-280.

Callan HG. 1986. Lampbrush chromosomes. Springer, Berlin.

Callan HG, Gall JG, Murphy C. 1991. Histone genes are located at the sphere loci of Xenopus lampbrush chromosomes. Chromosoma 101: 245-251.

Calvi BR, Lilly MA, Spradling AC. 1998. Cell cycle control of chorion gene amplification. Genes Dev 12: 734-744.

Clelland AK, Kinnear NP, Oram L, Burza J, Sleeman JE. 2009. The SMN protein is a key regulator of nuclear architecture in differentiating neuroblastoma cells. Traffic 10: 1585-1598.

Daneholt B. 2001. Packing and delivery of a genetic message. Chromosoma 110: 173-185.

Dej KJ, Spradling AC. 1999. The endocycle controls nurse cell polytene chromosome structure during Drosophila oogenesis. Development 126: 293-303.

Deryusheva S, Gall JG. 2009. Small Cajal body-specific RNAs of Drosophila function in the absence of Cajal bodies. Mol Biol Cell 20: 5250-5259.

DiMario PJ. 2004. Cell and molecular biology of nucleolar assembly and disassembly. Int Rev Cytol 239: 99-178. 
Doyle O, Corden JL, Murphy C, Gall JG. 2002. The distribution of RNA polymerase II largest subunit (RPB1) in the Xenopus germinal vesicle. J Struct Biol 140: 154-166.

Gall JG. 1968. Differential synthesis of the genes for ribosomal RNA during amphibian oogenesis. Proc Natl Acad Sci 60: 553-560.

Gall JG. 2003. The centennial of the Cajal body. Nat Rev Mol Cell Biol 4: 975-980.

Gall JG, Stephenson EC, Erba HP, Diaz MO, Barsacchi-Pilone G. 1981. Histone genes are located at the sphere loci of newt lampbrush chromosomes. Chromosoma 84: 159-171.

Gall JG, Diaz MO, Stephenson EC, Mahon KA. 1983. The transcription unit of lampbrush chromosomes. In Gene structure and regulation in development (ed. S Subtelny and F Kafatos), pp. 137-146. Liss, New York.

Gall JG, Bellini M, Wu Z, Murphy C. 1999. Assembly of the nuclear transcription and processing machinery: Cajal bodies (coiled bodies) and transcriptosomes. Mol Biol Cell 10: 43854402.

Jády BE, Darzacq X, Tucker KE, Matera AG, Bertrand E, Kiss T. 2003. Modification of Sm small nuclear RNAs occurs in the nucleoplasmic Cajal body following import from the cytoplasm. EMBO J 22: $1878-1888$.

Klingauf M, Stanek D, Neugebauer KM. 2006. Enhancement of U4/U6 small nuclear ribonucleoprotein particle association in Cajal bodies predicted by mathematical modeling. Mol Biol Cell 17: 4972-4981.

Lafarga M, Casafont I, Bengoechea R, Tapia O, Berciano MT 2009. Cajal's contribution to the knowledge of the neuronal cell nucleus. Chromosoma 118: 437-443.

Li CF, Pontes O, El-Shami M, Henderson IR, Bernatavichute YV, Chan SW, Lagrange T, Pikaard CS, Jacobsen SE. 2006. An ARGONAUTE4-containing nuclear processing center colocalized with Cajal bodies in Arabidopsis thaliana. Cell 126: 93 106.

Liu JL, Murphy C, Buszczak M, Clatterbuck S, Goodman R, Gall JG. 2006. The Drosophila melanogaster Cajal body. J Cell Biol 172: $875-884$.

Liu JL, Wu Z, Nizami Z, Deryusheva S, Rajendra TK, Gao H, Beumer KJ, Carroll D, Matera AG, Gall JG. 2009. Coilin is essential for Cajal body organization in Drosophila melanogaster. Mol Biol Cell 20: 1661-1670.

Marzluff WF. 2005. Metazoan replication-dependent histone mRNAs: A distinct set of RNA polymerase II transcripts. Curr Opin Cell Biol 17: 274-280.

Matera AG. 2006. Drosophila Cajal bodies: Accessories not included. J Cell Biol 172: 791-793.

Miller OL Jr, Beatty BR. 1969. Visualization of nucleolar genes. Science 164: 955-957.
Morgan GT, Doyle O, Murphy C, Gall JG. 2000. RNA polymerase II in Cajal bodies of amphibian oocytes. $J$ Struct Biol 129: 258268.

Morris GE. 2008. The Cajal body. Biochim Biophys Acta 1783: 2108-2115.

Murphy C, Wang Z, Roeder RG, Gall JG. 2002. RNA polymerase III in Cajal bodies and lampbrush chromosomes of the Xenopus oocyte nucleus. Mol Biol Cell 13: 3466-3476.

Raska I, Andrade LEC, Ochs RL, Chan EKL, Chang C-M, Roos G, Tan EM. 1991. Immunological and ultrastructural studies of the nuclear coiled body with autoimmune antibodies. Exp Cell Res 195: 27-37.

Raska I, Shaw PJ, Cmarko D. 2006. New insights into nucleolar architecture and activity. Int Rev Cytol 255: 177-235.

Stanek D, Neugebauer KM. 2006. The Cajal body: A meeting place for spliceosomal snRNPs in the nuclear maze. Chromosoma 115: 343-354.

Stein GS, Stein JL, Marzluff WF, Eds. 1984. Histone genes: Structure, organization, and regulation. Wiley, New York.

Stephenson EC, Erba HP, Gall JG. 1981. Histone gene clusters of the newt Notophthalmus are separated by long tracts of satellite DNA. Cell 24: 639-647.

Strzelecka M, Trowitzsch S, Weber G, Luhrmann R, Oates AC, Neugebauer KM. 2010. Coilin-dependent snRNP assembly is essential for zebrafish embryogenesis. Nat Struct Mol Biol 17: 403-409.

Tuma RS, Stolk JA, Roth MB. 1993. Identification and characterization of a sphere organelle protein. J Cell Biol 122: 767-773.

Tycowski KT, Shu MD, Kukoyi A, Steitz JA. 2009. A conserved WD40 protein binds the Cajal body localization signal of scaRNP particles. Mol Cell 34: 47-57.

Venteicher AS, Abreu EB, Meng Z, McCann KE, Terns RM, Veenstra TD, Terns MP, Artandi SE. 2009. A human telomerase holoenzyme protein required for Cajal body localization and telomere synthesis. Science 323: 644-648.

White AE, Leslie ME, Calvi BR, Marzluff WF, Duronio RJ. 2007. Developmental and cell cycle regulation of the Drosophila histone locus body. Mol Biol Cell 18: 2491-2502.

Wu C-HH, Gall JG. 1993. U7 small nuclear RNA in C snurposomes of the Xenopus germinal vesicle. Proc Natl Acad Sci 90: 6257-6259.

Wu Z, Murphy C, Gall JG. 1994. Human p80-coilin is targeted to sphere organelles in the amphibian germinal vesicle. Mol Biol Cell 5: 1119-1127.

Yang XC, Burch BD, Yan Y, Marzluff WF, Dominski Z. 2009. FLASH, a proapoptotic protein involved in activation of caspase-8, is essential for $3^{\prime}$ end processing of histone pre-mRNAs. Mol Cell 36: 267-278. 


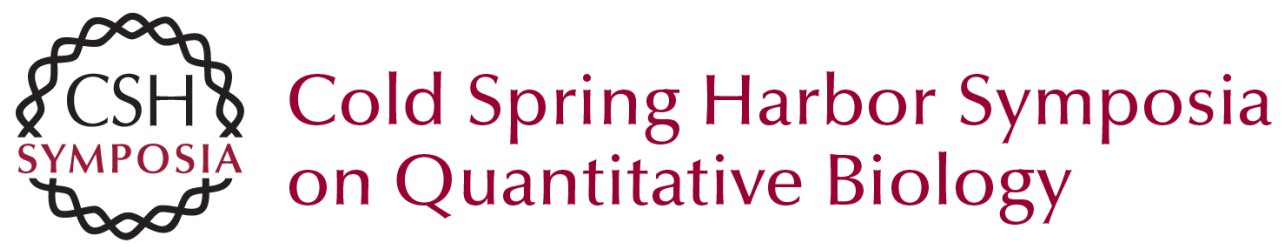

\title{
Cajal Bodies and Histone Locus Bodies in Drosophila and Xenopus
}

\author{
Z.F. Nizami, S. Deryusheva and J.G. Gall
}

Cold Spring Harb Symp Quant Biol 2010 75: 313-320 originally published online November 3, 2010 Access the most recent version at doi:10.1101/sqb.2010.75.005

References This article cites 42 articles, 22 of which can be accessed free at: http://symposium.cshlp.org/content/75/313.full.html\#ref-list-1

License

Email Alerting Receive free email alerts when new articles cite this article - sign up in the box at the Service top right corner of the article or click here.

To subscribe to Cold Spring Harbor Symposia on Quantitative Biology go to:

http://symposium.cshlp.org/subscriptions 